\title{
Desempenho muscular isocinético dos ombros em atletas de rúgbi
}

\author{
Isokinetic muscle performance of shoulders in rugby athletes
}

\author{
Gerson Saciloto Tadiello', Luiza Helena Brandelli Alves dos Santos', Taise Andreis Scopel', Bruno Soldatelli Zardo', \\ Vinícius Mazzochi Schmitt ${ }^{2}$, Leandro Viçosa Bonetti ${ }^{1} \bowtie$ \\ ${ }^{1}$ Curso de Fisioterapia da Universidade de Caxias do Sul (UCS). Caxias do Sul, RS \\ 2 Instituto de Medicina do Esporte e Ciências Aplicadas ao Movimento Humano, Laboratório de Análises Biomecânicas do Movimento Humano da UCS. \\ Caxias do Sul, RS
}

\section{RESUMO}

OBJETIVOS: Verificar a existência de assimetrias dos músculos rotadores externos e internos entre os dois ombros, em atletas amadores de rúgbi do gênero masculino.

MÉTODOS: Avaliações isocinéticas de atletas amadores do rúgbi masculino foram acessadas a partir do banco de dados do Instituto de Medicina do Esporte e Ciências Aplicadas ao Movimento Humano da Universidade de Caxias do Sul (IME-UCS). Foram incluídas as avaliações do ombro e excluídos os casos sem assinatura do Termo de Consentimento Livre e Esclarecido Institucional do IME-UCS prévia ao teste. O dinamômetro isocinético foi utilizado no modo concêntrico-concêntrico para os rotadores externos e internos dos músculos dos ombros nas velocidades angulares de $60 \% \mathrm{~s}$ e $180 \%$ s. Foram analisados os valores do pico de torque e da razão rotadores externos/internos dos ombros desses atletas. Para verificar a existência de assimetrias entre os dois lados do mesmo indivíduo, foi utilizado o teste T de Student e considerado o nível de significância de $<0,05$.

RESULTADOS: Foram analisados os dados de 12 atletas homens. Na análise do pico de torque na velocidade angular de $60 \%$, os valores médios da musculatura do membro dominante apresentaram-se significativamente superiores quando comparados ao não dominante: rotadores externos, $43,35 \pm 4,72 \mathrm{Nm}$ no membro dominante versus $38,92 \pm 4,22 \mathrm{Nm}$ no membro não dominante ( $\mathrm{p}=0,0012$ ); rotadores internos, $65,58 \pm 11,88 \mathrm{Nm}$ no membro dominante versus $61,69 \pm 11,98 \mathrm{Nm}$ no membro não dominante $(\mathrm{p}=0,0021)$. A $180^{\circ} \mathrm{s}$, os valores médios do membro não dominante apresentaram uma tendência a valores inferiores quando comparado ao membro dominante, mas sem significância estatística. Com relação aos valores médios da razão rotadores externos/internos, não foram encontradas diferenças estatisticamente significativa em nenhuma das velocidades angulares analisadas.

CONCLUSÕES: Os atletas amadores de rúgbi apresentaram um desempenho muscular inferior do membro não dominante quando comparado ao membro dominante na análise do pico de torque. Essa diferença não havia sido encontrada nos demais estudos com atletas de rúgbi e acreditamos que este resultado se justifique pela falta de treinamentos mais frequentes e adequados desses atletas amadores.

DESCRITORES: força muscular; ombro; atletas; rúgbi.

\section{ABSTRACT}

AIMS: To verify the existence of shoulder external and internal rotator muscle asymmetries in male amateur rugby athletes.

METHODS: Isokinetic evaluations of male amateur rugby athletes were accessed from the database of the Institute for Sports Medicine and Applied Sciences in Human Movement of Universidade de Caxias do Sul (IME-UCS). Shoulders evaluated were included in the study, whereas those cases without a free and informed consent form signed prior to the test were excluded. The isokinetic dynamometer was used in a concentric-concentric mode for the shoulder external and internal rotators at angular velocities of $60 \% \mathrm{~s}$ and $180 \%$ s. The peak torque and the external to internal rotator ratio were analyzed. Student's $t$ test and a significance level of $<0.05$ were used to verify the existence of asymmetries between the two sides of the same individual.

RESULTS: Data from 12 male athletes were analyzed. Regarding the peak torque at the angular velocity of $60^{\circ} / \mathrm{s}$, the mean muscle strength values of the dominant limb were significantly higher when compared to non-dominant limb: external rotators, $43.35 \pm 4.72 \mathrm{Nm}$ for the dominant limb $v s 38.92 \pm 4.22 \mathrm{Nm}$ for the non-dominant limb ( $\mathrm{p}=0.0012)$; internal rotators, $65.58 \pm 11.88 \mathrm{Nm}$ for the dominant limb $v s 61.69 \pm 11.98 \mathrm{Nm}$ for the non-dominant $\operatorname{limb}(\mathrm{p}=0.0021)$. At $180^{\circ} / \mathrm{s}$, the mean values of the non-dominant limb tended to be lower when compared to the dominant limb, without statistical significance. Regarding the mean values of the external to internal rotator ratio, no significant differences were found at any of the angular velocities analyzed.

CONCLUSIONS: Amateur rugby athletes showed lower muscle strength of the non-dominant limb when compared to the dominant limb in the peak torque measurements. This difference was not found in other studies with rugby athletes, and we believe that this result is justified by the lack of more frequent and adequate training of these amateur athletes.

KEY WORDS: muscle strength; shoulder; athletes; rugby. 
Abreviaturas: IME-UCS, Instituto de Medicina do Esporte e Ciências Aplicadas ao Movimento Humano da Universidade de Caxias do Sul; PT, pico de torque; RE, rotadores externos; RI, rotadores internos; razão RE/RI, razão rotadores externos/ internos; N/m, Newtons por metro.

\section{INTRODUÇÃO}

O rúgbi é um dos esportes mais praticados no mundo e com um número cada vez maior de praticantes no Brasil. Além do rúgbi caracterizar-se como um esporte de contato e de muita exigência física, os movimentos específicos como maul, ruck, scrum (formação ordenada) e tackle (placagem) aumentam significativamente o risco de lesões musculoesqueléticas durante os treinamentos e jogos [1-8]. Apesar das lesões na região da cabeça e dos membros inferiores ainda serem as mais comumente reportadas, alguns estudos têm relatado um aumento na frequência de lesões na articulação do ombro, tanto em atletas profissionais como amadores [4, 9-12].

A revisão de literatura de King et al. [2] demonstrou que o complexo articular do ombro é responsável por $28 \%$ das lesões musculoesqueléticas do rúgbi, enquanto a recente revisão de Kaux et al. [3] apresentou uma percentagem menor $(6,5 \%)$ de lesões nessa região. Em outra recente revisão de literatura, Papalia et al. [13] descrevem que as lesões no complexo articular do ombro estão relacionadas a severos danos aos tecidos moles que envolvem especificamente essa articulação, também denominada de glenoumeral. Problemas musculares também levam a instabilidades, aumentando ainda mais as chances de lesões nessa articulação durante a prática do rúgbi [4]. Geralmente, instabilidades em atletas de rúgbi ocorrem no membro não dominante (MND), devido às diferenças de força e coordenação do atleta, já que os passes realizados através de arremessos laterais resultam em maior força muscular e melhor coordenação no membro dominante (MD) $[14,15]$.

Considerando a importância da articulação do ombro durante a prática do rúgbi, o estudo do desempenho muscular dessa articulação é de grande importância para a identificação de fatores de risco, como desequilíbrios entre os membros e entre os músculos agonistas e antagonistas, ou identificação de fraquezas musculares. Sendo assim, a avaliação isocinética, por ser um método de avaliação de alta validade e confiabilidade, pode ser utilizada com o objetivo de verificar desempenho muscular desses atletas [16]. Apesar de vários estudos terem utilizado a avaliação isocinética no estudo muscular de diferentes articulações em diversas populações esportivas, há poucos estudos direcionados à articulação do ombro em atletas de rúgbi. Diante disto, o presente estudo teve como objetivo verificar, através de informações de um banco de dados, a existência de assimetrias nos músculos rotadores externos e internos entre os dois ombros em atletas amadores de rúgbi.

\section{MÉTODOS}

Este estudo, com delineamento transversal e retrospectivo, foi realizado no Instituto de Medicina do Esporte e Ciências Aplicadas ao Movimento Humano da Universidade de Caxias do Sul (IMEUCS) (Caxias do Sul, Rio Grande do Sul, Brasil). Este trabalho foi aprovado (protocolo 967.527) pelo Comitê de Ética em Pesquisa da Faculdade Cenecista Bento Gonçalves (Bento Gonçalves, Rio Grande do Sul, Brasil) e conduzido de acordo com as disposições legais da resolução no 466 do ano de 2012, do Conselho Nacional de Saúde, que aprova as diretrizes e normas regulamentadoras de pesquisas envolvendo seres humanos.

As informações do banco de dados do IME-UCS de avaliações isocinéticas da articulação do ombro de atletas do gênero masculino de uma equipe amadora de rúgbi da região da Serra Gaúcha fizeram parte da amostra deste estudo. O número amostral foi estabelecido por conveniência e, portanto, determinado de forma intencional e não probabilística, de acordo com o número de avaliações disponíveis. Foram incluídas as avaliações da articulação do ombro realizadas no modo concêntrico-concêntrico e foram excluídas as avaliações sem assinatura do Termo de Consentimento Livre e Esclarecido Institucional do IME-UCS prévia ao teste. Os atletas participavam de competições regionais e jogos amistosos, e os treinamentos técnicos e/ou táticos eram realizados três vezes por semana, totalizando 5 horas semanais.

As avaliações isocinéticas foram realizadas no dinamômetro isocinético do IME-UCS (Biodex System $4^{\circledR}$, Biodex Medical Systems, Shieley, Nova Iorque, EUA) e foram baseadas em um protocolo padronizado, onde todos os atletas primeiramente realizaram exercício de aquecimento em uma bicicleta ergométrica com velocidade moderada por 8 minutos. Após, os atletas foram encaminhados ao dinamômetro, posicionados em sedestação na cadeira, com o tronco inclinado a $85^{\circ}$ e estabilizados com cintos sobre o tronco e a pélvis para evitar movimentos compensatórios. O posicionamento do membro superior para a avaliação 
dos rotadores externos (RE) e rotadores internos (RI) do ombro foi de: $60^{\circ}$ de abdução do ombro no plano da escápula ( $30^{\circ}$ à frente do plano frontal) e $90^{\circ}$ de flexão do cotovelo; o punho foi estabilizado para evitar substituição de movimento e a amplitude de movimento foi limitada a $40^{\circ}$ de RE e $50^{\circ}$ de RI [17]. Os testes foram realizados primeiramente no $\mathrm{MD}$ e após no MND. O protocolo realizado durante o teste foi de cinco repetições máximas de RE e RI de ombro no modo concêntrico-concêntrico na velocidade angular de $60 \%$ e dez repetições na velocidade angular de $180 \%$ s, sendo que previamente ao teste em cada velocidade, os atletas executaram três repetições submáximas e uma máxima para fins de familiarização com os procedimentos, bem como para aquecimento. Foi estipulado o tempo de $1 \mathrm{mi}-$ nuto de descanso entre a avaliação em uma velocidade e outra, e o tempo de 5 minutos entre a avaliação de um membro e outro. Os atletas foram avaliados por um mesmo avaliador e foi utilizado comando verbal com o intuito de estimular o atleta, durante todo o teste, para a realização da sua máxima força.

Os valores de pico de torque (PT) e da razão rotadores externos/internos (razão RE/RI) da musculatura rotadora externa e interna dos ombros foram analisados estatisticamente no programa SPSS Statistics versão 17.0. Para verificar a normalidade da distribuição dos dados foi usado o teste de normalidade de Shapiro-Wilk. Os dados apresentaram distribuição normal e não paramétrica, e foram submetidos ao teste t de Student, sendo considerado o nível de significância de $<0,05$.

\section{RESULTADOS}

Fizeram parte do estudo 12 atletas do gênero masculino de rúgbi amador, que preencheram os critérios de inclusão e exclusão. A média de idade dos atletas era de 24,55 $\pm 3,20$ anos, a estatura média foi de $1,79 \pm 0,06 \mathrm{~m}$ e a massa corporal média foi de $92,27 \pm 14,98 \mathrm{~kg}$. Todos os atletas reportaram dominância de membro superior direito para os movimentos de jogo.

Os valores médios do PT da musculatura do ombro estão apresentados na Tabela 1. Na comparação entre o $\mathrm{MD}$ e o MND, podemos observar que os valores médios dos rotadores externos e internos do MND foram significativamente inferiores quando comparado aos do MD na velocidade angular de $60^{\circ} / \mathrm{s}$. A $180^{\circ} / \mathrm{s}$, o membro não dominante apresentou uma tendência a valores inferiores quando comparado ao membro dominante, mas essa diferença não foi estatisticamente significativa.

Tabela 1. Valores médios e desvio padrão do pico de torque da musculatura rotadora externa e rotadora interna da articulação do ombro do membro dominante e do membro não dominante, em atletas amadores de rúgbi masculino.

\begin{tabular}{|c|c|c|c|c|c|c|}
\hline \multirow{2}{*}{$\begin{array}{l}\text { Velocidades } \\
\text { angulares }\end{array}$} & \multicolumn{3}{|c|}{ Pico de torque dos rotadores externos $(\mathrm{Nm})$} & \multicolumn{3}{|c|}{ Pico de torque dos rotadores internos $(\mathrm{Nm})$} \\
\hline & $\begin{array}{c}\text { MD } \\
\text { Média } \pm \text { DP }\end{array}$ & $\begin{array}{c}\text { MND } \\
\text { Média } \pm \text { DP }\end{array}$ & $\mathbf{p}^{*}$ & $\begin{array}{c}\text { MD } \\
\text { Média } \pm \text { DP }\end{array}$ & $\begin{array}{c}\text { MND } \\
\text { Média } \pm \text { DP }\end{array}$ & $\mathbf{p}^{*}$ \\
\hline $60 \% / \mathrm{s}$ & $43,35 \pm 4,72$ & $38,92 \pm 4,22$ & 0,0012 & $65,58 \pm 11,88$ & $61,69 \pm 11,98$ & 0,0021 \\
\hline $180 \% / \mathrm{s}$ & $42,37 \pm 7,94$ & $40,62 \pm 9,23$ & 0,2700 & $59,80 \pm 8,98$ & $56,52 \pm 11,74$ & 0,1300 \\
\hline
\end{tabular}

$\mathrm{MD}$, membro dominante; MND, membro não dominante; $\mathrm{Nm}$, Newton por metro; DP, desvio padrão.

* Teste t de Student.

Tabela 2. Valores médios e desvio padrão da razão rotadores externos/internos da articulação do ombro do membro dominante e do membro não dominante, em atletas amadores de rúgbi masculino.

\begin{tabular}{|c|c|c|c|}
\hline \multirow{2}{*}{$\begin{array}{l}\text { Velocidades } \\
\text { angulares }\end{array}$} & \multicolumn{3}{|c|}{ Razão RE/RI (\%) } \\
\hline & $\begin{array}{c}\text { MD } \\
\text { Média } \pm \text { DP }\end{array}$ & $\begin{array}{c}\text { MND } \\
\text { Média } \pm \text { DP }\end{array}$ & $\mathbf{p}^{*}$ \\
\hline $60 \% / \mathrm{s}$ & $67,48 \pm 11,10$ & $64,74 \pm 11,39$ & 0,0670 \\
\hline $180 \% / \mathrm{s}$ & $71,62 \pm 13,96$ & $72,85 \pm 15,27$ & 0,7100 \\
\hline
\end{tabular}

Razão RE/RI, razão rotadores externos/internos; MD, membro dominante; MND, membro não dominante; DP, desvio padrão.

* Teste t de Student.
Na Tabela 2 estão descritos os valores médios da razão RE/RI, sendo que na comparação entre o MD e o MND não foi encontrada diferença estatisticamente significativa em nenhuma das velocidades angulares analisadas.

\section{DISCUSSÃO}

Este estudo teve como objetivo verificar a existência de assimetrias nos valores do PT dos rotadores externos e internos e da razão RE/RI entre as articulações dos ombros de atletas de rúgbi. 
Analisar a funcionalidade muscular tornou-se uma prática comum para a identificação de desequilíbrios musculares em atletas, pois através desta é possível o desenvolvimento de programas de fortalecimento específicos que poderão culminar na diminuição de lesões musculoesqueléticas. A dinamometria isocinética é considerada o padrão ouro para avaliação do desempenho da musculatura esquelética, devido à sua reprodutibilidade e confiabilidade [18]. Os resultados do presente estudo não demonstraram diferenças estatisticamente significativas na velocidade angular de $180^{\circ} / \mathrm{s}$, entretanto a $60^{\circ} \%$ s o MD apresentou valores médios significativamente superiores ao do MND, tanto para a musculatura RE como da RI na análise do PT. Estudos isocinéticos com atletas de modalidades esportivas assimétricas, como voleibol [17, 19-23], handebol [24, 25], tênis [26-28], basebol [29, 30] e polo aquático [31] também demonstraram que o MD apresenta valores maiores de PT dos RE e/ou RI. A utilização preferencial de um dos membros durante os principais movimentos de uma determinada prática esportiva faz com que haja um aumento da força muscular desse membro. No entanto, as diferenças entre o MD e o MND encontradas neste estudo não foram encontradas em outros estudos com atletas de rúgbi [32, 33]. Foram encontrados na literatura publicada apenas dois estudos sobre análise do PT do ombro de atletas de rúgbi: Edouard et al. [32] e McDonough e Funk [33]. Realizados com metodologias semelhantes às do presente estudo, demonstraram que a dominância não interfere na força muscular dos rotadores do ombro. Contudo, devemos considerar que os treinamentos mais frequentes e adequados dos atletas semiprofissionais e profissionais avaliados nos citados estudos podem justificar o equilíbrio muscular não encontrado nos atletas amadores [32, 33].

Para atletas de rúgbi, o bom desempenho muscular dos RE e RI do ombro é de extrema importância para a prevenção de lesões desse complexo articular, uma das regiões anatômicas mais acometidas por lesões e que, juntamente com a articulação do joelho, corresponde aos maiores prejuízos aos atletas [4]. Especificamente para os atletas avaliados neste estudo, a fraqueza do MND é ainda mais preocupante, pois durante a prática do rúgbi esse membro é mais predisposto a lesões quando comparado ao $\mathrm{MD}$, tanto lesões traumáticas como não traumáticas [14]. O desequilíbrio de força entre os membros está relacionado a um risco maior de lesões não traumáticas do ombro; entretanto esta relação não está clara no que se refere às lesões traumáticas [32].
A maioria das lesões traumáticas do ombro ocorrem durante o tackle [34-37], que é o fundamento técnico utilizado para derrubar ou impedir o avanço do adversário que está com a posse da bola [38]. Esse gesto esportivo é realizado a partir de uma posição agachada com os braços abduzidos para buscar combater o tronco do oponente, resultando em um impacto anteroposterior nos ombros e braços [37]. O tackle é responsável por 50 a $85 \%$ das lesões do ombro. Lacerações do manguito rotador, lesões de Bankart, SLAP (Superior Labrum Anterior to Posterior Lesion, ou seja, lesão do lábio superior de anterior para posterior) e luxações são as consequências mais comuns dessa manobra [13, 38-40]. Além disso, o MND é o mais atingido porque os atletas protegem-se com esse membro enquanto carregam a bola com o MD [34]. Devido a isso, exercícios de fortalecimento dos rotadores do $\mathrm{MND}$, que tenham o objetivo reestabelecer a força muscular desse membro, podem melhorar a estabilização da articulação glenoumeral e, consequentemente, auxiliar na prevenção de lesões traumáticas durante os impactos relacionados ao tackle.

Na comparação dos valores médios do PT entre os RI e RE, foi possível observar que os atletas avaliados apresentaram a musculatura RI com valores médios superiores ao dos RE, em ambos os membros e em ambas as velocidades angulares avaliadas. Os demais estudos com atletas de rúgbi demonstraram resultados similares [32, 33, 41]. O maior PT dos RI é um resultado esperado, tanto para sujeitos saudáveis como para atletas praticantes de qualquer modalidade esportiva devido à musculatura RI ser mais numerosa, anatomicamente maior e mais forte [42], além de ter um braço de alavanca maior quando comparado aos RE [43]. No entanto, a mais importante relação entre os RE e RI se refere aos valores razão do PT entre esses grupos musculares, comumente chamada de razão RE/RI ou razão agonista/antagonista. Vários estudos têm usado os valores dessa razão para descrever o equilíbrio muscular da articulação do ombro, e consequentemente, identificar o risco de lesões durante a prática esportiva [44-46].

Além disso, o estudo da razão RE/RI serve como um guia para o diagnóstico, terapêutica e reabilitação de lesões do ombro. A comparação entre os membros e a análise dos valores da razão RE/RI são informações importantes tanto para a avaliação do desempenho muscular como na prevenção de lesões, pois através da identificação de desequilíbrios musculares é possível realizar uma proposta de intervenção prévia [41, 45]. Entretanto, os resultados do presente estudo não 
demonstraram diferenças estatisticamente significativas entre os membros na analise dos valores médios da razão RE/RI, mesmo a $60^{\circ} / \mathrm{s}$, onde o MND apresentou valores de PT significativamente inferiores ao MD. Como os valores do PT do MND foram inferiores tanto para a musculatura RE como para RI, a razão não foi afetada significativamente.

Além da comparação entre os membros, a análise dos valores percentuais deve ser considerada e esta deve estar entre $60 \%$ e $75 \%$ para sujeitos do gênero masculino [44]. Os resultados deste estudo demonstraram valores médios entre os valores recomendados pela literatura, demonstrando que esses atletas apresentavam um equilíbrio de força entre os RE e RI da articulação do ombro. Os resultados dos estudos de Edouard et al. [32] e de McDonough e Funk [33] também não mostraram diferenças estatisticamente significativas, e os valores estavam dentro dos sugeridos pela literatura, corroborando com a assertiva de que a prática do rúgbi não tem efeito sobre o equilíbrio de forças entre os rotadores do ombro. Entretanto, Bolton et al. [41] encontraram diferenças estatisticamente significativas entre os membros na análise dos valores médios da razão RE/RI e valores percentuais fora dos parâmetros de normalidade durante a avaliação de atletas semiprofissionais de rúgbi a $60 \%$ s. Entretanto, os próprios autores não consideram claros os reais motivos destes resultados [41].
Como limitações deste estudo, podemos destacar o pequeno número amostral, o número reduzido de velocidades angulares avaliadas, assim como a avaliação apenas no modo concêntrico. Além disso, por se tratar de um estudo de análise de banco de dados, não foi possível obter algumas informações importantes sobre lesões musculoesqueléticas prévias ou envolvimento em outras atividades físicas. Apesar dessas limitações, o estudo alcançou resultados importantes, sendo que o mais relevante foi a identificação de um desempenho muscular inferior do MND quando comparado ao $\mathrm{MD}$, em atletas amadores de rúgbi masculino. Essa diferença não havia sido encontrada nos demais estudos com atletas de rúgbi. Acreditamos que este resultado se justifique pela falta de treinamentos mais frequentes e adequados dos atletas amadores. Devido a isso, um enfoque preventivo através do fortalecimento muscular deve ser realizado/orientado a esses atletas, pois o MND tem ainda um maior risco de lesões quando comparado ao MD.

\section{NOTAS}

\section{Apoio financeiro}

Este estudo não recebeu apoio financeiro de fontes externas.

Declaração de conflito de interesses

Os autores declaram não haver conflitos de interesses relevantes ao conteúdo deste estudo, informam ter tido acesso a todos os dados obtidos e assumem completa responsabilidade pela integridade dos resultados.

\section{REFERÊNCIAS}

1. Kaplan KM, Goodwillie A, Strauss EJ, Rosen JE. Rugby injuries: a review of concepts and current literature. Bull NYU Hosp Jt Dis. 2008;66(2):86-93.

2. King DA, Hume PA, Milburn PD, Guttenbeil D. Match and training injuries in rugby league: a review of published studies. Sports Med. 2010 Feb 1;40(2):163-78. https://doi.org/10.2165/11319740-000000000-00000

3. Kaux J-F, Julia M, Delvaux F, Croisier J-L, Forthomme B, Monnot D, Chupin M, Crielaard J-L, Le Goff C, Durez P, Ernst P, Guns S, Laly A. Epidemiological review of injuries in Rugby Union. Sports. 2015;3(1):21-9. https://doi.org/10.3390/sports3010021

4. Palmer-Green DS, Stokes KA, Fuller CW, England M, Kemp SP, Trewartha G. Training activities and injuries in English youth academy and schools Rugby Union. Am J Sports Med. 2015;43(2):475-81. https://doi.org/10.1177/0363546514560337

5. Austin D, Gabbett T, Jenkins D. The physical demands of Super 14 Rugby Union. J Sci Med Sport. 2011;14(3):259-63. https://doi. org/10.1016/j.jsams.2011.01.003

6. Williams S, Trewartha G, Kemp S, Stokes K. A meta-analysis of injuries in senior men's professional Rugby Union. Sports Med. 2013;43(10):1043-55. https://doi.org/10.1007/s40279-013-0078-1

7. Macqueen AE, Dexter WW. Injury trends and prevention in Rugby Union football. Curr Sports Med Rep. 2010;9(3):139-43. https:// doi.org/10.1249/JSR.0b013e3181df124c

8. Pearce CJ, Brooks JH, Kemp SP, Calder JD. The epidemiology of foot injuries in professional Rugby Union players. Foot Ankle Surg. 2011 Sept;17(3):113-8. https://doi.org/10.1016/j.fas.2010.02.004

9. Palmer-Green DS, Stokes KA, Fuller CW, England M, Kemp SP, Trewartha G. Match injuries in English youth academy and schools Rugby Union: an epidemiological study. Am J Sports Med. 2013 Apr;41(4):749-55. https://doi.org/10.1177/0363546512473818

10. King DA, Gabbett TJ. Injuries in the New Zealand semiprofessional rugby league competition. NZ J Sports Med. 2009;36(1):6-15.

11. Haseler CM, Carmont MR, England M. The epidemiology of injuries in English youth community Rugby Union. Br J Sports Med. 2010;44(5):1093-9. https://doi.org/10.1136/bjsm.2010.074021 
12. Usman J, McIntosh AS. Upper limb injury in rugby union football: results of a cohort study. Br J Sports Med. 2013;47(6):374-9. https://doi.org/10.1136/bjsports-2012-091224

13. Papalia R, Tecame A, Torre G, Narbona P, Maffulli N, Denaro V. Rugby and shoulder trauma: A systematic review. Transl Med UniSa. 2014 Sept 1;12:5-13.

14. Sundaram A, Bokor DJ, Davidson AS. Rugby Union on-field position and its relationship to shoulder injury leading to anterior reconstruction for instability. J Sci Med Sport. 2011 Mar;14(2):111-4. https://doi.org/10.1016/j.jsams.2010.08.005

15. Gabbett TJ. Incidence of injuries in junior and senior rugby league players. J Sci Med Sport. 2008 June;11(3):323-8. https://doi. org/10.1016/j.jsams.2007.06.003

16. Bruyèrea O, Beaudart C, Reginster J-Y, Buckinx F, Schoene D, Hirani VM, Cooper C, Kanis JA, Rizzoli R, McCloskey E, Cederholm T, Cruz-Jentoft A, Freiberger E. Assessment of muscle mass, muscle strength and physical performance in clinical practice: An international survey. Eur Geriatr Med. 2016;7(3):243-6. https://doi.org/10.1016/j.eurger.2015.12.009

17. Mendonça LDM, Bittencourt NFN, Anjos MTS, Silva AA, Fonseca ST. Avaliação muscular isocinética da articulação do ombro em atletas da seleção brasileira de voleibol sub-19 e sub-21 masculino. Rev Bras Med Esporte. 2010;16(2):107-11. https://doi.org/10.1590/ S1517-86922010000200006

18. Caruso JF, Brown LE, Tufano JJ. The reproducibility of isokinetic dynamometry data. Isokinet Exerc Sci. 2012;20(4):239-53. https:// doi.org/10.3233/IES-2012-0477

19. Wang HK, Macfarlane A, Cochrane T. Isokinetic performance and shoulder mobility in elite volleyball athletes from the United Kingdom. Br J Sports Med. 2000;34(1):39-43. https://doi.org/10.1136/bjsm.34.1.39

20. Michael J, Konig D, Hessling U, Popken F, Eysel P. Results of shoulder isokinetic testing in volleyball players. Sportverletz Sportschaden. 2003 June; 17(2):71-4.

21. Markou S, Vagenas G. Multivariate isokinetic asymmetry of the knee and shoulder in elite volleyball players. Eur J Sport Sci 2006;6(1):71-80. https://doi.org/10.1080/17461390500533147

22. Hadzic V, Sattler T, Veselko M, Markovic G, Dervisevic E. Strength asymmetry of the shoulders in elite volleyball players. J Athl Train. 2014;49(3):338-44. https://doi.org/10.4085/1062-6050-49.2.05

23. Franceschini KC, Nissola N, Zardo BS, Tadielo GS, Bonetti LV. Isokinetic performance of shoulder external and internal rotators in adolescent male volleyball athletes. Int Archi Med. 2016;9(1):1-7. https://doi.org/10.3823/2011

24. Andrade M dos S, Fleury AM, de Lira CA, Dubas JP, da Silva AC. Profile of isokinetic eccentric-to-concentric strength ratios of shoulder rotator muscles in elite female team handball players. J Sports Sci. 2010;28(7):743-9. https://doi.org/10.1080/02640411003645687

25. Andrade M dos S, de Lira CA, Vancini RL, de Almeida AA, Benedito-Silva AA, da Silva AC. Profiling the isokinetic shoulder rotator muscle strength in 13- to 36-year-old male and female handball players. Phys Ther Sport. 2013;14(4):246-52. https://doi.org/10.1016/j. ptsp.2012.12.002

26. Ellenbecker T, Roetert EP. Age specific isokinetic glenohumeral internal and external rotation strength in elite junior tennis players. J Sci Med Sport. 2003;6(1):63-70. https://doi.org/10.1016/S1440-2440(03)80009-9

27. Gozlan G, Bensoussan L, Coudreuse JM, Fondarai J, Gremeaux V, Viton JM, Delarque A. Isokinetic dynamometer measurement of shoulder rotational strength in healthy elite athletes (swimming, volley-ball, tennis): Comparison between dominant and nondominant shoulder. Ann Readapt Med Phys. 2006;49(1):8-15. https://doi.org/10.1016/j.annrmp.2005.07.001

28. Saccol MF, Gracitelli GC, da Silva RT, Laurino CF, Fleury AM, Andrade M dos S, da Silva AC. Shoulder functional ratio in elite junior tennis players. Phys Ther Sport. 2010;11(1):8-11. https://doi.org/10.1016/j.ptsp.2009.11.002

29. Noffal GJ. Isokinetic eccentric-to-concentric strength ratios of the shoulder rotator muscles in throwers and nonthrowers. Am J Sports Med. 2003;31(4):537-41.

30. Hurd WJ, Kaplan KM, ElAttrache NS, Jobe FW, Morrey BF, Kaufman KR. A profile of glenohumeral internal and external rotation motion in the uninjured high school baseball pitcher, part II: strength. J Athl Train. 2011;46(3):289-95. https://doi.org/10.4085/1062$6050-46.3 .289$

31. Campos TF, Petrone KCO, Navega MT, Renner AF, Mattiello-Rosa SM. Estudo dos picos de torque concêntricos e excêntricos dos rotadores mediais e laterais do ombro de atletas do pólo aquático. Rev Bras Fisioter. 2005;9(2):137-43.

32. Edouard P, Frize N, Calmels P, Samozino P, Garet M, Degache F. Influence of rugby practice on shoulder internal and external rotators strength. Int J Sports Med. 2009;30(12):863-7. https://doi.org/10.1055/s-0029-1237391

33. McDonough A, Funk L. Can glenohumeral joint isokinetic strength and range of movement predict injury in professional rugby league. Phys Ther Sport. 2014;15(2):91-6. https://doi.org/10.1016/j.ptsp.2013.07.001

34. Bandeira F, Neves EB, Moura MAM, Nohama P. A termografia no apoio ao diagnóstico de lesão muscular no esporte. Rev Bras Med Esporte. 2014;20(1):59-64. https://doi.org/10.1590/S1517-86922014000100012

35. Brooks JHM, Fuller CW, Kemp SPT, Reddin DB. Epidemiology of injuries in English Professional Rugby Union: Part II match injuries. Brit J Sport Med. 2005;39(10):767-75. https://doi.org/10.1136/bjsm.2005.018408

36. McIntosh AS, Savage TN, McCrory P, Fréchède BO, Wolfe R. Tackle characteristics and injury in a cross section of rugby union football. Med Sci Sports Exerc. 2010;42(5):977-84. https://doi.org/10.1249/MSS.0b013e3181c07b5b

37. Helgeson K, Stoneman P. Shoulder injuries in rugby players: mechanisms, examination, and rehabilitation. Phys Ther Sport. 2014;15(4):218-27. https://doi.org/10.1016/j.ptsp.2014.06.001

38. Gabbett TJ. Incidence of injury in semi-professional rugby league players. Br J Sports Med. 2003;37(1):36-44. https://doi.org/10.1136/ bjsm.37.1.36 
39. Crichton J, Jones DR, Funk L. Mechanisms of traumatic shoulder injury in elite rugby player. Br J Sports Med. 2012;46(7):538-42. https://doi.org/10.1136/bjsports-2011-090688

40. Horsley IG, Pearson J, Green A, Rolf C. A comparison of the musculoskeletal assessments of the shoulder girdles of professional rugby players and professional soccer players. Sports Med Arthrosc Rehabil Ther Technol. 2012 Sept 10;4(1):32. https://doi.org/10.1186/17582555-4-32

41. Bolton G, Moss SJ, Sparks M, Venter PC. Thoracic posture, shoulder muscle activation patterns and isokinetic strength of semiprofessional rugby union players. S Afr J SM. 2013;25(1):12-7. https://doi.org/10.17159/2078-516X/2013/v25i1a386

42. Dark A, Ginn KA, Halaki M. Shoulder muscle recruitment patterns during commonly used rotator cuff exercises: an electromyographic study. Phys Ther. 2007;87(8):1039-46. https://doi.org/10.2522/ptj.20060068

43. Lin HT, Nakamura Y, Su FC, Hashimoto J, Nobuhara K, Chao EY. Use of virtual, interactive, musculoskeletal system (VIMS) in modeling and analysis of shoulder throwing activity. J Biomech Eng. 2005;127(3):525-30. https://doi.org/10.1115/1.1894387

44. Ellenbecker TS, Davies GJ. The application of isokinetics in testing and rehabilitation of the shoulder complex. J Athl Train. 2000 July;35(3):338-50.

45. Codine P, Bernard PL, Pocholle M, Herisson C. Isokinetic strength measurement and training of the shoulder: methodology and results. Ann Readapt Med Phys. 2005 Mar;48(2):80-92. https://doi.org/10.1016/j.annrmp.2004.07.002

46. Wilk KE, Obma P, Simpson CD, Cain EL, Dugas JR, Andrews JR. Shoulder injuries in the overhead athlete. J Orthop Sports Phys Ther. 2009 Feb;39(2):38-54. https://doi.org/10.2519/jospt.2009.2929 